\title{
Fatores que influenciam a adesão às medidas de precaução padrão e de contato no cuidado à pacientes críticos: Revisão integrativa
}

\author{
Factors influencing adherence to standard and contact precautionary measures in care for critical \\ patients: Integrative review
}

Factores que influencian la adhesión a las medidas de precaución estándar y contacto en la atención de pacientes críticos: Revisión integrativa

Recebido: 25/03/2021 | Revisado: 03/04/2021 | Aceito: 10/04/2021 | Publicado: 20/04/2021

Patrícia Miranda Sá

ORCID: https://orcid.org/0000-0002-0479-2477 Universidade Federal do Estado do Rio de Janeiro, Brasil E-mail: pat.mirandasa@gmail.com

Caroline Gomes Marambaia

ORCID: https://orcid.org/0000-0003-4648-0540 Universidade Federal do Estado do Rio de Janeiro, Brasil E-mail: carolinemarambaia@hotmail.com Priscila Carvalho de Souza

ORCID: https://orcid.org/0000-0001-9507-4878 Universidade Federal do Estado do Rio de Janeiro, Brasil E-mail: pri.ufrj.micro@gmail.com

Aline Affonso Luna

ORCID: https://orcid.org/0000-0002-7648-8634 Universidade Federal do Estado do Rio de Janeiro, Brasil E-mail: aline.luna@unirio.br

Natália Chantal Magalhães da Silva

ORCID: https://orcid.org/0000-0003-1883-4313 Universidade Federal do Estado do Rio de Janeiro, Brasil E-mail: natalia.c.silva@unirio.br

\begin{abstract}
Resumo
Objetivo: Identificar, na literatura científica, os fatores que influenciam a adesão de medidas de precaução padrão e de contato por profissionais de saúde durante o cuidado à pacientes críticos. Metodologia: Trata-se de uma revisão integrativa da literatura, baseada em investigações científicas publicadas entre 2015 e 2020. Foram efetuadas buscas nas bases de dados: BDENF, LILACS, MEDLINE, PUBMED e SCIELO, a partir dos descritores: Precauções Universais; Unidades de Terapia Intensiva; e, Controle de Infecções. Resultados: Foram identificadas 1.522 publicações, dos quais 10 atingiram os critérios de elegibilidade estabelecidos. A partir dos resultados, identificou-se que a baixa adesão às precauções padrão e de contato podem estar relacionadas aos fatores individuais dos profissionais, à deficiência na formação, à estrutura organizacional da instituição, ao gerenciamento da unidade e às condições de trabalho. Considerações Finais: As taxas de adesão às medidas de precaução padrão e de contato pelos profissionais da saúde atuantes nas Unidades de Terapia Intensiva, ainda estão aquém do esperado, seja no quesito higienização das mãos, manejo e descarte de perfurocortantes ou uso de equipamentos de proteção individual.
\end{abstract}

Palavras-chave: Precauções universais; Unidades de terapia intensiva; Controle de infecções.

\begin{abstract}
Objective: To identify, in the scientific literature, the factors that influence the adherence to standard and contact precautionary measures by health professionals during the care of critical patients. Methodology: This is an integrative literature review, based on scientific investigations published between 2015 to 2020. Searches were carried out in databases: Foram efetuadas buscas nas bases de dados: BDENF, LILACS, MEDLINE, PUBMED and SCIELO, using the descriptors: Universal Precautions; Intensive Care Units; and, Infection Control. Results: 1,522 publications were identified, of which 10 met the established eligibility criteria. From the results, it was identified that the low adherence to standard and contact precautions may be related to the individual factors of the professionals, the deficiency in training, the organizational structure of the institution, the management of the unit and the working conditions. Final Considerations: The rates of adherence to standard and contact precautionary measures by health professionals working in the Intensive Care Units are still below expectations, whether in terms of hand hygiene, handling and disposal of sharps or use of personal protective equipment.
\end{abstract}

Keywords: Universal precautions; Intensive care units; Infection control. 


\begin{abstract}
Resumen
Objetivo: Identificar, en la literatura científica, los factores que influencian en el cumplimiento de las medidas cautelares estándar y de contacto de los profesionales de la salud durante la atención de pacientes críticos. Metodología: Se trata de una revisión de integrativa de la literatura, basada en investigaciones científicas publicadas entre 2015 y 2020. Se realizaron búsquedas en las bases de datos: BDENF, LILACS, MEDLINE, PUBMED y SCIELO, utilizando los descriptores: Precauciones Universales; Unidades de Cuidados Intensivos; y Control de Infecciones. Resultados: Se identificaron 1.522 publicaciones, de las cuales 10 cumplieron con los criterios de elegibilidad establecidos. A partir de los resultados, se identificó que la baja adherencia a las normas y precauciones de contacto puede estar relacionada con los factores individuales de los profesionales, la deficiencia en la formación, la estructura organizativa de la institución, la gestión de la unidad y las condiciones de trabajo. Consideraciones Finales: Los índices de adherencia a las medidas de precaución estándar y contacto de los profesionales de la salud que trabajan en las Unidades de Cuidados Intensivos, aún se encuentran por debajo de las expectativas, ya sea en términos de higiene de manos, manejo y eliminación de objetos punzantes o uso de equipos de protección individual.
\end{abstract}

Palabras clave: Precauciones universales; Unidades de cuidados intensivos; Control de infecciones.

\title{
1. Introdução
}

As infecções relacionadas à assistência à saúde (IRAS) são denominadas pela Organização Mundial de Saúde (OMS) como infecções adquiridas pelo paciente durante sua estadia em um hospital ou em outro serviço de saúde e que não se encontravam presentes, nem sequer em incubação, no momento de sua admissão (World Health Organization [WHO], 2016). Em certos casos, são caracterizadas como eventos adversos (EA), uma vez que podem ocorrer por meio de procedimentos (diagnósticos ou terapêuticos) executados por profissionais da área da saúde, tanto em ambiente hospitalar, ambulatorial ou em domicílio (Gil et al., 2018).

Segundo a OMS, anualmente, 1,4 milhões de indivíduos contraem infecções. Na Europa, em média 6,8\% dos pacientes internados adquirem, no mínimo, uma IRAS. Já em países em desenvolvimento, o risco é até quatro vezes maior (Silva et al., 2018). Atualmente, em países desenvolvidos, calcula-se que a cada 100 pacientes internados em instituições assistenciais pelo menos sete irão adquirir IRAS, e, em países em desenvolvimento, esse número sobe para dez (Castro \& Rodrigues, 2019).

Essas infecções aumentam de maneira considerável os custos institucionais necessários para a prestação dos cuidados ao paciente, além disso, eleva o tempo de internação, a morbimortalidade e a disseminação de microrganismos multirresistentes (Agência Nacional de Vigilância Sanitária [ANVISA], 2017).

Como principais fontes de disseminação de infecções em unidades assistenciais estão o uso demasiado e indiscriminado de antimicrobianos, a falta de aderência às medidas básicas de controle como a higienização de mãos, dispositivos/equipamentos e pacientes imunocomprometidos (Barros, 2017).

Dentre essas, as Unidades de Terapia Intensiva (UTI) são vistas como principais locais de ocorrência e disseminação das IRAS, com uma incidência $20 \%$ superior a outros setores de atendimento. Isso se deve ao fato dessas unidades serem uma das mais complexas do hospital, com um perfil de pacientes gravemente enfermos, múltiplos procedimentos e dispositivos invasivos, tempo elevado de internação, polifarmácia, grande período de contato do profissional com o paciente, exposição prolongada à antimicrobianos e colonização por microrganismos multirresistentes (Silva et al., 2018).

Como as IRAS, em sua maioria, são consideradas complicações preveníveis e estão associadas à resistência bacteriana nas unidades de cuidados aos pacientes, a implementação de estratégias focadas na precaução e isolamento de patógenos são capazes de reduzir as taxas de infecção, funcionando como uma barreira para a transmissão de um microrganismo entre pacientes, tanto de forma direta, quanto indireta (Melo, 2019; Faro, 2016).

Nesse âmbito, os profissionais da saúde desempenham papel fundamental, visto que podem servir de transporte para esses microrganismos. Segundo Barros et al. (2019) o profissional de saúde que se contamina ao entrar em contato com o paciente, com objetos do mesmo ou até mesmo com as superfícies inanimadas do ambiente hospitalar poderá causar a 
transmissão cruzada de microrganismos, se não forem utilizadas as medidas de precaução adequadas. Portanto, de forma a prevenir possíveis infecções, estes devem desempenhar suas funções de maneira segura e com responsabilidade (Faro, 2016; Siegel et al., 2019).

Visando prevenir a transmissão de infecções durante o atendimento ao paciente e proteger o profissional contra risco ocupacional, existem as chamadas medidas de precaução universais, dentre as quais estão às medidas de precaução padrão (PP) e as precauções especiais, que se dividem em três categorias: precauções de contato, para aerossóis e para gotículas (Centers for Disease Control and Prevention [CDC], 2016; Castro \& Rodrigues, 2019).

As PP são classificadas como um conjunto de práticas de prevenção às infecções, com uso indicado para a assistência de todos os pacientes, independente da suspeita ou confirmação da presença de um agente infecioso. Incluem: higienização das mãos, uso correto de equipamento de proteção individual (EPI) e manejo seguro de perfurocortantes. Já as precauções especiais, são utilizadas quando as vias de transmissão do agente infecioso não são interrompidas com o uso das PP (Faria et al., 2019; Porto \& Marziale, 2016; Castro \& Rodrigues, 2019).

Dentre as precauções especiais, a precaução de contato acaba por ser mais relevante nas UTI. Isso se dá pelo fato desse ambiente servir como um reservatório frequente de microrganismos multirresistentes, visto que os pacientes ali internados se encontram mais suscetíveis a infecções, em decorrência da condição clínica sensível em que se encontram, da exposição à procedimentos invasivos e da administração intensa de antimicrobianos (Basso et al., 2016; Reis et al., 2015).

Logo, considerando a relevância e a magnitude que as IRAS têm no nosso país e a importância da criação de uma cultura de segurança, o presente estudo teve como objetivo: identificar, na literatura científica, os fatores que influenciam a adesão de medidas de precaução padrão e de contato por profissionais de saúde durante o cuidado a pacientes críticos.

\section{Metodologia}

Trata-se de um estudo bibliográfico do tipo revisão integrativa de artigos indexados nas bases de dados: Base de Dados em Enfermagem (BDENF), Literatura Latino-Americana e do Caribe em Ciências da Saúde (LILACS), Medical Literature Analysis and Retrievel System on-line (MEDLINE), Public Medline (PUBMED) e a Scientific Eletronic Library Online (SCIELO).

O presente estudo foi fundamentado em cinco etapas metodológicas seguindo o referencial teórico de Whittemore e Knafl (2005): 1) identificação do tema e seleção da questão de pesquisa; 2) Busca dos artigos nas bases de dados e estabelecimento de critérios para inclusão e exclusão; 3) Avaliação dos achados da pesquisa; 4) Análise e intepretação dos resultados; 5) apresentação de uma síntese dos artigos selecionados.

Assim sendo, a partir da definição do tema, foi elencada a seguinte questão norteadora: "Quais os fatores que influenciam a adesão de medidas de precaução padrão e de contato por profissionais de saúde durante o cuidado à pacientes críticos?"

Para a seleção dos descritores (DeCs/MeSh) e/ou palavras-chave a serem utilizadas nesta revisão, foi realizada uma pesquisa prévia nas bases de dados elegidas, selecionando, assim, três descritores: "Precauções Universais", "Unidades de Terapia Intensiva" e "Controle de Infecções” e, suas variações em inglês e espanhol. Além dos descritores, optou-se pelo uso da palavra-chave "Precaução de contato" e suas variantes em inglês e espanhol, visto que ao utilizar somente o descritor "Precauções Universais" apresentaram-se resultados escassos para estudos que abordassem a precaução de contato, ampliandose estes quando a palavra-chave esteve presente.

Os critérios para inclusão dos estudos nessa revisão foram: possuir disponibilidade eletrônica na íntegra de forma gratuita; ter sido publicado nos idiomas inglês, português ou espanhol, no período de 2015 a 2020; e, possuir relação com a temática proposta nesta revisão. Já os critérios de exclusão foram: repetições nas bases de dados; dissertações, teses, trabalhos 
de conclusão de curso, manuais, artigos de opinião, editoriais, estudos de casos, cartas ao editor, notas prévias e resumos de eventos.

A coleta de dados foi desenvolvida no mês de junho de 2020. Os registros identificados foram submetidos a dois processos de filtragem. Na primeira filtragem, por meio do programa EndNote ${ }^{\circledR}$, foram excluídas as duplicadas e aplicados os critérios de inclusão estabelecidos. Os dados coletados foram organizados em planilhas no programa Microsoft Excel ${ }^{\circledR}$, visando facilitar a visualização dos mesmos durante a desenvolvimento dos resultados e discussão. O processo de análise dos estudos se deu por meio da leitura incessante do material, observando criteriosamente sua adequação aos critérios metodológicos previamente estabelecidos e sua correlação com o objetivo proposto por esta revisão.

Com relação aos aspectos éticos, não houve nenhuma alteração do conteúdo original dos artigos em prol de benefícios às autoras ou à esta revisão, respeitando-se e mantendo seus direitos autorais.

Os estudos foram classificados de acordo com os níveis hierárquicos de evidência propostos pelo Centre for Evidence-Based Medicine (2009) de Oxford, ao qual são divididos em: nível 1A - Revisões sistemáticas e meta-análises de ensaios clínicos comparáveis. Estudos randomizados controlados com desfecho clínico relevante e bem delineados; Nível 1B Ensaios clínicos randomizados e controlados, com estreito intervalo de confiança; Nível 1C - Estudos do tipo "tudo ou nada" e estudos de série de casos controlados; Nível 2A - Revisão sistemática, homogênea, de estudos de coorte; Nível 2B - Estudos de coorte, incluindo os com baixa qualidade de randomização, sem acompanhamento longo ou controle e estudos transversais; Nível 2C - Estudos ecológicos e Resultados de pesquisas (estudos de observação de resultados terapêuticos ou evolução clínica); Nível 3A - Revisão sistemática, homogênea, de estudos de caso-controle; Nível 3B - Estudos de caso-controle; Nível 4 - Relato de casos, incluindo estudos de coorte ou caso-controle de baixa qualidade; Nível 5 - Opinião de especialistas ou de autoridades respeitadas sem avaliação crítica, estudos com animais ou estudos fisiológicos e revisões literárias não sistemáticas (Pedrosa et al., 2015).

A partir do exposto, foi formulado um fluxograma (Figura 1) descrevendo o processo de buscas nas bases de dados. 
Figura 1 - Fluxograma indicativo do resultado das buscas nas bases de dados.
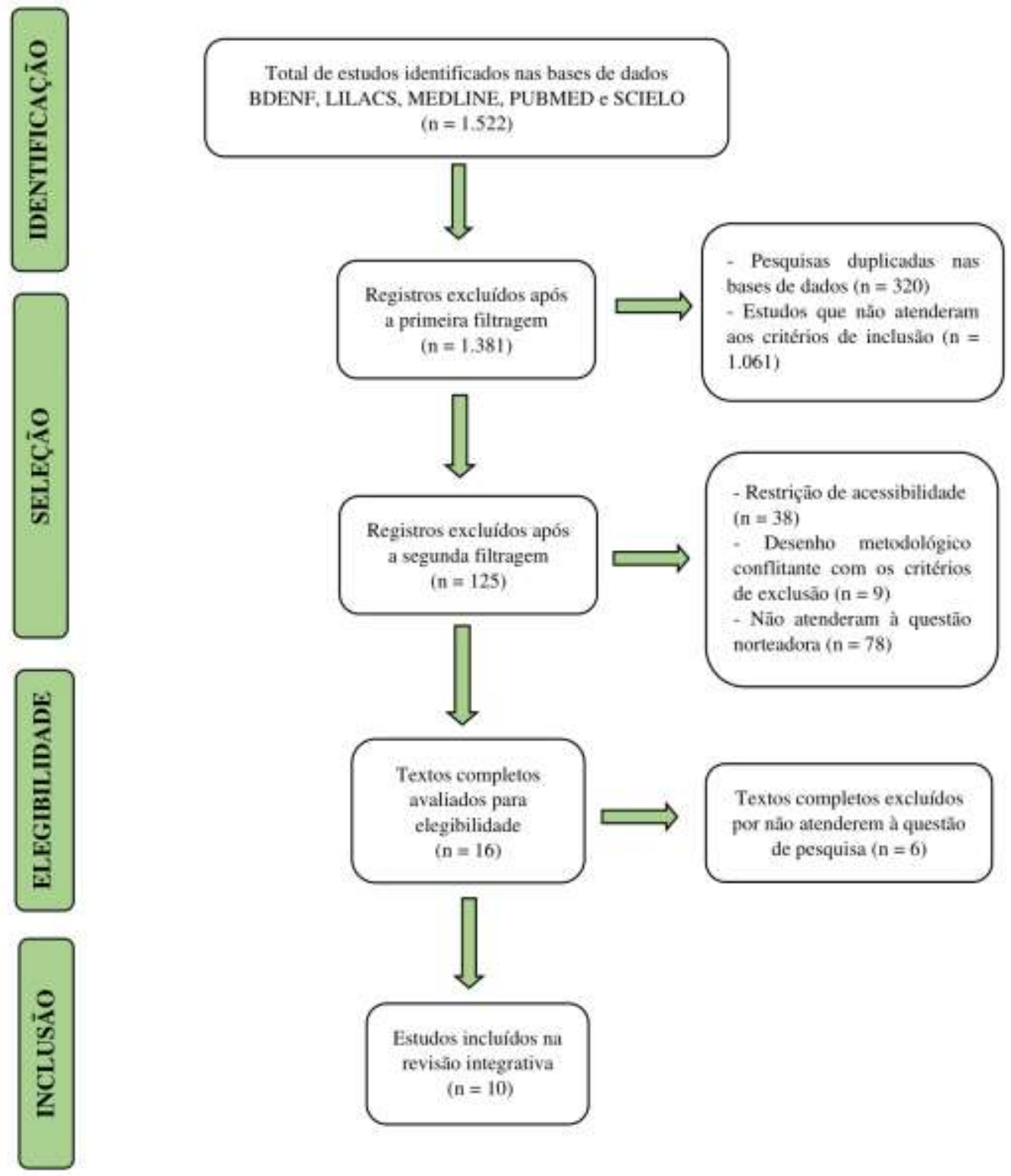

Fonte: Adaptado de PRISMA (Moher et al., 2009).

\section{Resultados}

Para cumprir as fases metodológicas dessa revisão integrativa, 10 artigos foram considerados.

Quanto à caracterização dos estudos selecionados, tem-se que três possuíam abordagem qualitativa e sete abordagem quantitativa. Os artigos tiveram como ano majoritário de publicação o ano de 2019, seguidos dos anos de 2018 e 2017 , ambos com duas publicações, e os anos de 2016 e 2015, cada um com uma publicação. As pesquisas foram desenvolvidas no Brasil (7), na Nigéria (2) e no Canadá/Brasil (1). O idioma predominante foi o português (7), seguido do inglês (3). Com relação ao nível de evidencia, um dos estudos se enquadrava no nível IIA, sete no nível IV, e dois no nível V. O Quadro 1 apresenta a síntese dos dados obtidos, descrevendo os estudos perante ano de publicação, autoria, título, objetivo e nível de evidência. 
Quadro 1 - Síntese dos artigos selecionados para compor a revisão integrativa. Rio de Janeiro, RJ, Brasil, 2020.

\begin{tabular}{|c|c|c|c|c|}
\hline Ano & Autoria & Título & Objetivo & $\begin{array}{l}\text { Nível de } \\
\text { Evidência }\end{array}$ \\
\hline 2019 & Castro e Rodrigues. & $\begin{array}{l}\text { Auditoria de práticas de precauções- } \\
\text { padrão e contato em Unidade de } \\
\text { Terapia Intensiva. }\end{array}$ & $\begin{array}{l}\text { Avaliar a estrutura e a adesão às medidas de } \\
\text { precauções-padrão e específicas dos profissionais } \\
\text { de saúde em Unidade de Terapia Intensiva de } \\
\text { hospital de ensino, no Distrito Federal. }\end{array}$ & IV \\
\hline 2019 & Faria et al. & $\begin{array}{l}\text { Conhecimento e adesão do } \\
\text { enfermeiro às precauções padrão em } \\
\text { unidades críticas. }\end{array}$ & $\begin{array}{l}\text { Identificar o conhecimento e a adesão de } \\
\text { enfermeiros às precauções padrão em unidades de } \\
\text { atendimento a pacientes críticos. }\end{array}$ & IV \\
\hline 2019 & Barros et al. & $\begin{array}{l}\text { Controle de infecções a pacientes } \\
\text { em precaução de contato. }\end{array}$ & $\begin{array}{l}\text { Descrever o conhecimento do enfermeiro sobre as } \\
\text { medidas de precaução de contato. }\end{array}$ & IV \\
\hline 2019 & Boeira et al. & $\begin{array}{l}\text { Controle de infecções e medidas de } \\
\text { segurança do paciente abordados em } \\
\text { projetos pedagógicos da } \\
\text { enfermagem. }\end{array}$ & $\begin{array}{l}\text { Caracterizar o ensino acerca da segurança do } \\
\text { paciente e das medidas de precauções-padrão para } \\
\text { prevenção e controle de infecções, nos cursos de } \\
\text { graduação em enfermagem. }\end{array}$ & $\mathrm{V}$ \\
\hline 2018 & Moralejo et al. & $\begin{array}{l}\text { Improving adherence to Standard } \\
\text { Precautions for the control of health } \\
\text { care-associated infections. }\end{array}$ & $\begin{array}{l}\text { Avaliar a eficácia das intervenções voltadas aos } \\
\text { profissionais de saúde para melhorar a adesão às } \\
\text { Precauções Padrão no atendimento ao paciente. }\end{array}$ & IIA \\
\hline 2018 & Adegboye et al. & $\begin{array}{l}\text { Knowledge, awareness and practice } \\
\text { of infection control by health care } \\
\text { workers in the intensive care units } \\
\text { of a tertiary hospital in Nigeria. }\end{array}$ & $\begin{array}{l}\text { Avaliar o conhecimento, consciência e prática de } \\
\text { controle de infecção, entre os funcionários da UTI } \\
\text { de um hospital terciário na Nigéria. }\end{array}$ & IV \\
\hline 2017 & Alvim e Gazzinelli. & $\begin{array}{l}\text { Conhecimento dos profissionais de } \\
\text { enfermagem em relação às medidas } \\
\text { de prevenção das infecções. }\end{array}$ & $\begin{array}{l}\text { Avaliar o conhecimento dos profissionais de } \\
\text { enfermagem em relação às medidas de prevenção } \\
\text { das infecções relacionadas à assistência à saúde. }\end{array}$ & IV \\
\hline 2017 & Ndu e Arinze-Onyia. & $\begin{array}{l}\text { Standard precaution knowledge and } \\
\text { adherence: Do Doctors differ from } \\
\text { Medical Laboratory Scientists? }\end{array}$ & $\begin{array}{l}\text { Comparar a adesão e conhecimento das } \\
\text { precauções padrão (PP) entre cientistas de } \\
\text { laboratórios médicos (MLSs) e médicos. }\end{array}$ & IV \\
\hline 2016 & Porto e Marziale. & $\begin{array}{l}\text { Motivos e consequências da baixa } \\
\text { adesão às precauções padrão pela } \\
\text { equipe de enfermagem. }\end{array}$ & $\begin{array}{l}\text { Analisar os motivos e as consequências da baixa } \\
\text { adesão às PP pela equipe de enfermagem. }\end{array}$ & $\mathrm{V}$ \\
\hline 2015 & Piai-Morais et al. & $\begin{array}{l}\text { Fatores que influenciam a adesão às } \\
\text { precauções-padrão entre } \\
\text { profissionais de enfermagem em } \\
\text { hospital psiquiátrico }\end{array}$ & $\begin{array}{l}\text { Avaliar e correlacionar fatores individuais, } \\
\text { relativos ao trabalho e organizacionais, que } \\
\text { influenciam a adesão às precauções-padrão entre } \\
\text { profissionais de enfermagem de hospital } \\
\text { psiquiátrico do interior paulista. }\end{array}$ & IV \\
\hline
\end{tabular}

Fonte: Autores.

Dentre os estudos selecionados, seis são focados na equipe de Enfermagem, três tem por foco a equipe multiprofissional, e um, a equipe médica. Ademais, estes possuem abordagens distintas, sendo seis artigos sobre a correlação entre o conhecimento e adesão dos profissionais às medidas de precaução ( 03 de enfermagem, 02 multiprofissionais e 01 sobre a equipe médica), dois são sobre os fatores de influência entre os profissionais de Enfermagem, um é sobre o ensino na graduação de Enfermagem, e, por fim, um dos estudos tem por foco a gestão na UTI, avaliando a estrutura e a adesão das 
medidas de precaução, com foco multiprofissional.

Vale ressaltar que dos três artigos pautados na equipe multiprofissional, um tem por participantes médicos, residentes, enfermeiros e fisioterapeutas (Piai-Morais et al., 2015), outro é composto por técnicos de enfermagem, enfermeiros, médicos e fisioterapeutas (Castro \& Rodrigues, 2019), e, no terceiro, não são especificados os indivíduos claramente (Moralejo et al., 2018).

Assim sendo, foram identificados como fatores que influenciam a adesão dos profissionais às medidas de precaução padrão e de contato: a falta de conscientização, mitos e crenças por parte dos profissionais, baixa percepção de risco, descuido/esquecimento, jornadas excessivas, ritmo intenso de trabalho, falta de tempo para vestir os EPIs, ausência de recursos adequados, plantões noturnos, desgaste físico e emocional, equipes reduzidas, excesso de confiança, estresse, falta ou insuficiência de insumos, baixo compromisso da gestão hospitalar, má qualidade dos equipamentos de proteção individual, armazenamento em local distante do ponto de assistência, desconforto ao uso ou irritação cutânea, deficiente de capacitação técnica e carência de treinamentos (Porto \& Marziale, 2016; Piai-Morais et al., 2015; Castro \& Rodrigues, 2019; Barros et al., 2019; Boeira et al., 2019; Alvim \& Gazzinelli, 2017; Moralejo et al., 2018; Ndu \& Arinze-Onyia, 2017).

\section{Discussão}

Após análise dos estudos incluídos na revisão integrativa, pode-se observar que de forma majoritária a adesão às medidas de precaução, tanto padrão, quanto de contato encontram-se aquém do desejado, apresentando índices que variam de médios à baixos, além de um alto índice de usos inadequados dos EPIs quando necessário. Assim, com base nesses índices, os estudos trouxeram possíveis motivações para as taxas abaixo da média esperada e estratégias para melhora-las (Porto \& Marziale, 2016; Moralejo et al., 2018; Faria et al., 2019; Barros et al., 2019).

Em geral, os achados se correlacionam com o fato de que dentre as principais causas de disseminação de infecções associadas à assistência está à falta de aderência às medidas básicas de precaução, sobretudo a higienização de mãos e, que os profissionais de saúde desempenham um papel de relevância no transporte desses microrganismos (Faro, 2016; Siegel et al., 2019).

$\mathrm{Na}$ Nigéria, uma pesquisa envolvendo 80 profissionais, apontou que apesar de 80,3\% dos participantes terem por conhecimento o fato das mãos serem o veículo mais comum de transmissão de infecções, 32,5\% desses profissionais possuíam uma prática ineficiente de higienização das mesmas e, somente, 51,2\% calçavam novas luvas antes de examinar outros pacientes (Adegboye et al., 2018).

Em contraponto, um estudo transversal realizado em uma UTI e uma Unidade de Pacientes Críticos (UCP), com 40 enfermeiros, verificou que $80 \%$ deles sempre realizavam a higienização das mãos no intervalo entre a prestação de cuidados a diferentes pacientes, $90 \%$ após retirada das luvas e $100 \%$ após contato com materiais biológicos potencialmente contaminados (Faria et al, 2019). No entanto, apesar dos altos índices, os resultados ainda se encontram abaixo do esperado, visto que esse deveria ser um procedimento criteriosamente realizado por todos os profissionais.

Diante desta circunstância, a ANVISA $(2017,2018)$ ressalta que para prevenir as IRAS causadas por transmissão cruzadas, as mãos devem ser higienizadas, com produto apropriado, em cinco momentos considerados necessários e essenciais, sendo eles: antes de tocar o paciente; anteriormente a realização de procedimentos limpos/assépticos; após risco de exposição à fluidos corporais; após contato com o paciente; e após contato com superfícies próximas ao mesmo, reforçando que o uso de luvas não substitui a necessidade de higiene das mãos.

Ao analisarem a taxa de adesão ao uso de luvas, o estudo de Faria et al. (2019) indicou que apesar de 97,5\% dos enfermeiros aderirem ao uso de luvas para realização de procedimentos que apresentassem possibilidade de contato com materiais biológicos potencialmente contaminados, a não utilização desse EPI por parte dos profissionais ao realizarem certos 
procedimentos chegou a atingir: 32,5\% ao aplicar injeções intramusculares ou subcutâneas, 12,5\% ao manusear amostras de sangue, $10,0 \%$ ao realizar punções venosas e 5,0\% ao realizar procedimentos que envolvam a possibilidade de contato com a mucosa do paciente. Esses dados se tornam ainda mais preocupantes quando agregados ao percentual de profissionais que não utilizavam os demais EPIs ao realizarem procedimentos com possibilidade de contato com fluidos corporais, secreções, excreções ou respingos de sangue, já que 82,5\% não faziam uso de gorros e propés descartáveis, 70,0\% não utilizavam óculos de proteção, 55,0\% não usavam aventais de proteção e 15,0\% não aderiam ao uso de máscaras (Faria et al., 2019).

Por ter como foco as medidas de precaução padrão, o estudo ainda traz dados sobre o manejo de perfurocortantes, demonstrando que apenas 47,5\% adotavam o comportamento de não reencapar agulhas usadas, enquanto o manejo e descarte dos mesmos eram realizados de forma correta por 100\% dos profissionais (Faria et al., 2019). Corroborando com o achado, um estudo realizado na Nigéria com 143 médicos e 136 patologistas clínicos, apontou que 63,3\% dos médicos e 55,1\% dos patologistas ainda reencapam as agulhas antes de descarta-las (Ndu \& Arinze-Onyia, 2017). Apesar da relevância dessa medida para prevenção de infecções e acidentes laborais, informações sobre a adesão da mesma foram explicitadas somente em três de oito estudos ao qual tinham como foco, total ou parcial, as medidas de precaução padrão.

Uma auditoria realizada com 52 profissionais que atuavam na assistência direta a pacientes internados em uma UTI apontou que a média da taxa de adesão ao uso de EPIs quando indicado foi de 72,72\%. Ao analisarem a taxa de uso de forma correta ou incorreta dos quatro EPI juntos (luvas, avental, máscara e óculos), as taxas variaram de 29,79\% a 50,19\%. Já a média de taxa de acerto ao analisar conjuntamente o percentual de adesão aos quatro EPIs, conforme precaução que o paciente estava submetido, foi somente de 46,97\% (Castro \& Rodrigues, 2019).

Mediante a taxa de adesão individual dos quatro EPIs, o mesmo estudo (Castro \& Rodrigues, 2019) revelou que a adesão às luvas e avental foi alta, atingindo os $91 \%$, porém frisando que a maioria dos pacientes se encontrava em precaução de contato. A adesão ao uso de máscaras também foi alta, alcançando os $80 \%$. Os óculos, no entanto, foram o EPI mais negligenciado, com somente $24,56 \%$ de uso quando indicados e, principalmente em procedimentos de aspiração endotraqueal, que, no entanto, não representou nem $5 \%$ do total de uso para esse procedimento em específico. Ressalta-se que apesar de detalhar as taxas correlatas ao uso de EPI, esse estudo não apresenta os índices de adesão a outras medidas, como as de higienização de mãos ou manejo de perfurocortantes.

Vale ressaltar que, de acordo com a ANVISA (2004), as medidas de precaução padrão devem ser utilizadas, pelos profissionais, para o cuidado de todos os pacientes independente da presença ou ausência de doenças infecciosas comprovadas. Devendo, portanto, incluir: o uso completo, e não parcial, do EPI (luvas, máscaras, avental e óculos de proteção), a higienização das mãos, o manuseio e descarte adequado de perfurocortantes, a limpeza e desinfecção ou esterilização de artigos e equipamentos de assistência ao paciente, os cuidados com o ambiente e roupas contaminadas, bem como o uso de quarto privativo em casos específicos, buscando adaptar essas medidas conforme confirmação etiológica.

Examinando os motivos para a baixa adesão das medidas de precaução, sobretudo padrão, pode-se observar que vários se correlacionam com a insuficiência ou falta de investimento nas condições de trabalho, que além de expor os profissionais e pacientes a riscos, pode gerar um clima de insatisfação, desmotivação e desarmonia no ambiente de trabalho (Porto \& Marziale, 2016).

O desconhecimento dos profissionais de saúde, também é visto como um dos fatores que podem explicar os baixos índices de adesão. Estudos demonstram que esse fato pode ter correlação, tanto com a formação profissional, visto que por vezes são apontadas lacunas nos conteúdos curriculares e na prática docente, quanto com a carência de ações educativas ofertadas pelas instituições de trabalho, dificultando a construção de uma base de conhecimento sólida para a prevenção e controle de infeções (Boeira et al., 2019; Alvim \& Gazzinelli, 2017).

A existência de uma lacuna entre o conhecimento e a prática pode ser observada, por exemplo, nos baixos índices de 
higienização das mãos, indicando que apesar de essa ser considerada a principal medida de prevenção e controle de infecções e de existir, nos últimos anos, diversos estudos, guidelines e protocolos estruturados orientando sobre sua importância e técnica correta, ainda há dúvidas e um certo distanciamento por parte dos profissionais.

Esta afirmativa pode ser observada em um estudo de análise documental realizada em seis cursos de graduação em Enfermagem, o qual aponta que das 39 disciplinas dos cursos participantes apenas 15 (38,5\%) abordavam o tópico de higienização das mãos. Assim, os resultados revelam que apesar desta ser uma medida para prevenção de infecções inerente a qualquer cuidado, ainda é pouco explorada nos processos de ensino-aprendizagem dos cursos de nível superior, fortalecendo, assim, a explicação para a baixa adesão dos profissionais a essa medida (Boeira et al., 2019).

Outro apontamento para a existência dessa lacuna está associado a fatores individuais dos profissionais, como a autoconfiança gerada pela vasta experiência profissional e desatualização sobre os conteúdos atuais publicados na literatura científica, gerando uma falsa noção de que sua destreza e experiência podem substituir as medidas de proteção indicadas (Alvim \& Gazzinelli, 2017; Faria et al., 2019). Esse fato atrelado à carência de treinamentos específicos e frequentes pelas instituições de trabalho dificulta a obtenção de êxito, pelos profissionais, nos processos que circundam a prevenção e controle de infecções (Alvim \& Gazzinelli, 2017).

No entanto, se inexiste uma relação entre a atitude na prática e o conhecimento, também não se pode esperar que os profissionais tomassem atitudes adequadas sem o devido (re) conhecimento dos riscos a que se encontram expostos e do uso correto dos diferentes EPIs, reforçando, assim, a necessidade de investir em ações educativas e treinamentos para todos os profissionais da saúde (Faria et al., 2019; Moralejo et al., 2018). Deste modo, a implementação de estratégias educacionais se torna fundamental para reduzir a exposição ocupacional, minimizar a ocorrência de acidentes laborais e garantir a segurança dos profissionais e pacientes.

Em corroboração com os achados de Barros et al. (2019), Adegboye et al. (2018), Ndu e Arinze-Onyia (2017), o Ministério da Saúde aponta que a baixa adesão as medidas de higienização das mãos, vão além dos quesitos educacionais, estando comumente associada com a frequência com que os profissionais da saúde necessitam lavar suas mãos, com a tendência que os produtos utilizados no processo têm de causar ressecamento e/ou irritação da pele, e com o acesso dos profissionais aos equipamentos e insumos necessários (ANVISA, 2009).

Na revisão sistemática de Moralejo et al. (2018), o uso e distribuição adequadas de soluções alcoólicas foi apontada como estratégia para melhorar os índices de adesão às medidas de higienização das mãos. Esse levantamento é reiterado pelo Ministério da Saúde que considera seu uso uma opção mais efetiva à higienização das mãos do que quando comparado com o uso de sabonetes comuns ou aqueles associados à antissépticos (ANVISA, 2009).

No entanto, de acordo com a ANVISA (2018), o forte e desagradável odor das soluções alcoólicas e a sensação de mãos pegajosas logo após seu uso, são fatores que dificultam a aderência aos cinco momentos para higienização das mãos nos serviços de saúde. Além disso, essa barreira pode ser intensificada se seguida do uso de luvas de procedimento com pó, visto que a substancia ao entrar em contato com o álcool forma um resíduo indesejável nas mãos.

Ademais, os estudos levantados também apontaram como fatores facilitadores para a inadequação no uso de medidas de precaução padrão e de contato: seu uso indiscriminado, a sobrecarga de trabalho e a urgência dos serviços (Barros et al., 2019; Piai-morais et al., 2015). Em unidades de cuidado a pacientes críticos, a sobrecarga de trabalho e a necessidade de agir rapidamente em muitas situações, se tornam grandes obstáculos para a adesão às precauções, já que os profissionais consideram antes de tudo os cuidados aos pacientes e, posteriormente, a sua própria segurança (Faria et al., 2019; Piai-morais et al., 2015).

Nesse âmbito, a gestão da instituição de saúde possui papel fundamental, visto que esta é responsável pela manutenção do local de trabalho, permitindo reduzir obstáculos e incentivar o profissional frente à tomada de decisão da 
própria proteção (Piai-morais et al., 2015). Desta forma, estudos apontam que o baixo comprometimento da gestão hospitalar para o fornecimento de recursos estruturais básicos, materiais e insumos adequados, como os equipamentos de proteção individual, se torna uma barreira à adesão às medidas de precaução (Ndu \& Arinze-Onyia, 2017).

A baixa adesão as medidas de precaução trazem consequências, tanto para os profissionais e pacientes, quanto para as organizações, em decorrência da aquisição de infecções nosocomiais, acidentes laborais e prejuízos institucionais (Porto \& Marziale, 2016). Com base nisso, observa-se que todos os artigos analisados recomendaram intervenções que possibilitem aumentar a adesão às medidas de precaução padrão e de contato. A maioria recomendou a implementação de práticas pedagógicas no âmbito de trabalho, estimulando sua abordagem por meio de práticas inovadoras, como o uso de tecnologias e das metodologias ativas de ensino, proporcionando espaço para que os profissionais exponham suas experiências e reflexões sobre seu processo de trabalho, para assim identificar as possíveis motivações que os levam a não aderirem as medidas de precaução indicadas e planejar ações voltadas à redução desses obstáculos (Porto \& Marziale, 2016; Moralejo et al., 2018; Faria et al., 2019; Barros et al., 2019).

Para a ANVISA (2004), o controle e prevenção de infecções dependem fundamentalmente das medidas administrativas e das medidas de proteção individual. Assim, os treinamentos oferecidos aos profissionais devem ser pautados nos dados epidemiológicos, na transmissibilidade da doença, no risco ocupacional, nas normas de precaução e isolamento, no uso correto dos EPI, nos indicadores de saúde e metas para melhorias do serviço (ANVISA, 2016).

De modo a ratificar as estratégias de combate as barreiras encontradas, a OMS destaca que para se alcançar uma alta taxa de adesão às medidas de precaução, sobretudo, no quesito higienização das mãos, os profissionais de saúde precisam de: programas de educação; treinamento; observação; feedback; diretrizes de ensino claras; incentivos financeiros; elogios dos superiores, de modo a se sentirem valorizados; bem como advertências em caso de baixo desempenho; suporte administrativo; participação ativa em nível institucional; pias automatizadas; desenvolvimento de percepção de risco; e de produtos de higiene das mãos adequados e de fácil acesso (WHO, 2009).

Logo, como os fatores de influência para a adesão as medidas de precaução padrão e de contato extrapolam a área de abrangência das práticas educativas, necessitam-se ser adotadas estratégias que integrem um conjunto de elementos, como a cultura organizacional, a organização do trabalho vigente, a valorização dos profissionais, as condições de trabalho oferecidas e o modelo de gestão (Porto \& Marziale, 2016).

\section{Considerações Finais}

Pode-se verificar, por meio desta revisão, que as taxas de adesão às medidas de precaução padrão e de contato pelos profissionais de saúde atuantes nas UTI, ainda se encontram muito aquém do esperado.

Os motivos da baixa adesão às precauções padrão e de contato relacionam-se, sobretudo, aos fatores individuais dos profissionais, as deficiências na formação, a estrutura organizacional de instituição, problemas correlatos ao gerenciamento da unidade e as condições de trabalho. Ademais, vale destacar a lacuna teórico-prática encontrada, no que diz respeito a principal e melhor medida de prevenção e controle de infecções: a higienização das mãos. Essa reforçada pelo baixo uso e/ou pelo uso errôneo dos equipamentos de proteção individual, além de expor desnecessariamente o profissional a materiais potencialmente contaminados, facilita a ocorrência de infecções cruzadas.

Desta forma, mediante o papel que os profissionais da saúde desempenham no transporte de microrganismos, ressaltase a necessidade e a importância da gestão hospitalar em implementar estratégias educacionais continuadas e permanentes nos serviços de Terapia Intensiva e incentivar a participação dos trabalhadores, visando, assim, reduzir a exposição ocupacional, minimizar a ocorrência de acidentes laborais e garantir a segurança dos profissionais e pacientes.

Salienta-se, portanto, que a revisão integrativa cumpriu com o seu objetivo, permitindo-se contribuir para o incentivo 
e adesão dos profissionais às medidas de precaução padrão e de contato nas Unidades de Terapia Intensiva, ao sistematizar e sintetizar o conhecimento e as estratégias que vem sendo empregadas nas literaturas cientificas nos últimos anos.

A revisão possui por limitação a escassez de publicações encontradas, sobretudo, com foco nas medidas de precaução de contato, no entanto, as evidências levantadas podem ser úteis para discussões sobre a temática, assim como para o desenvolvimento de futuras pesquisas que abordem a adesão às medidas de precaução padrão e de contato no cuidado a pacientes críticos e, que poderão vir a somar ou refutar os achados deste estudo.

\section{Referências}

Adegboye, M. B., Zakari, S., Ahmed, B. A., \& Olufemi, G. H. (2018). Knowledge, awareness and practice of infection control by health care workers in the intensive care units of a tertiary hospital in Nigeria. African health sciences, 18(1), 72-78. https://doi.org/10.4314/ahs.v18i1.11

Alvim, A. L. S., \& Gazzinelli, A. (2016). Conhecimento dos profissionais de enfermagem em relação às medidas de prevenção das infecções. Revista de Enfermagem UFPE on line, 11(1), 18-23. https://periodicos.ufpe.br/revistas/revistaenfermagem/article/view/11873

Agência Nacional de Vigilância Sanitária. (2004). Curso Infecção Relacionada à Assistência à Saúde - IRAS. Módulo 5: Risco Ocupacional e Medidas de $\begin{array}{lllll}\text { Precauçes } & e & \text { Isolamento. } & \text { Versão } & \text { 1.0. }\end{array}$ $\% 20$ Risco\%20Ocupacional\%20e\%20Medidas\%20de\%20Precau\%E7\%F5es\%20e\%20Isolamento.pdf

Agência Nacional de Vigilância Sanitária. (2009). Segurança do Paciente em Serviços de Saúde: Higienização das Mãos. https://bvsms.saude.gov.br/bvs/publicacoes/seguranca_paciente_servicos_saude_higienizacao_maos.pdf

Agência Nacional de Vigilância Sanitária. (2016). Programa Nacional de Prevenção e Controle de Infecções Relacionadas à Assistência à Saúde (20162020). https://www20.anvisa.gov.br/segurancadopaciente/index.php/publicacoes/item/pnpciras-2016-2020

Agência Nacional de Vigilância Sanitária. (2017). Medidas de Prevenção de Infecção Relacionada à Assistência à Saúde. https://www20.anvisa.gov.br/segurancadopaciente/index.php/publicacoes/item/caderno-5

Agência Nacional de Vigilância Sanitária. (2018). Nota Técnica $n^{o}$ 01/2018 GVIMS/GGTES/ANVISA: Orientações gerais para higiene das mãos em serviços de saúde. https://www20.anvisa.gov.br/segurancadopaciente/index.php/alertas/item/nota-tecnica-n-01-2018-gvims-ggtes-anvisa-orientacoes-gerais-parahigiene-das-maos-em-servicos-de-saude

Barros, F. E. (2017). Construindo estratégia educativa relacionada a infecções na assistência à saúde em unidade hematológica e a enfermagem [Dissertação Mestrado, Universidade Federal do Estado do Rio de Janeiro]. Hórus: Repositório Institucional da UNIRIO. http://www.repositoriobc.unirio.br:8080/xmlui/handle/unirio/10969?show=full

Barros, F. E., Soares, E., Teixeira, M. L. O., \& Branco, E. M. S. C. (2019). Controle de infecções a pacientes em precaução de contato. Revista de Enfermagem UFPE on line, 13(4), 1081-1089. https://periodicos.ufpe.br/revistas/revistaenfermagem/article/view/238991.

Basso, M. E., Pulcinelli, R. S. R., Aquino, A. R. C., \& Santos, K. F. (2016). Prevalência de infecções bacterianas em pacientes internados em uma unidade de terapia intensiva (UTI). Revista Brasileira de Análises Clínicas, 48(4), 383-388. https://doi.org/10.21877/2448-3877.201600307

Boeira, E. R., Silva e Souza, A. C., Pereira, M. S., Vila, V. S. C., \& Tipple, A. F. V. (2019). Controle de infecções e medidas de segurança do paciente abordados em projetos pedagógicos da enfermagem. Revista da Escola de Enfermagem da USP, 53, e03420. https://doi.org/10.1590/s1980$220 \times 2017042303420$

Castro, A. F., \& Rodrigues, M. C. S. (2019). Auditoria de práticas de precauções-padrão e contato em Unidade de Terapia Intensiva. Revista da Escola de Enfermagem da USP, 53, e3508. https://doi.org/10.1590/s1980-220x2018018603508

Centers for Disease Control and Prevention. (2016). Guide to infection prevention for outpatient settings: minimum expectations for safe care. https://www.cdc.gov/infectioncontrol/pdf/outpatient/guide.pdf

Centre for Evidence-Based Medicine. (2009). Oxford Centre for Evidence-based Medicine - Levels of Evidence. https://www.cebm.net/2009/06/oxford-centreevidence-based-medicine-levels-evidence-march-2009/

Faria, L. B. G., Santos, C. T. B., Faustino, A. M., Oliveira, L. M. A. C., \& Cruz, K. C. T. (2019). Conhecimento e adesão do enfermeiro às precauções padrão em unidades críticas. Texto \& Contexto - Enfermagem, 28, e20180144. https://doi.org/10.1590/1980-265x-tce-2018-0144

Faro, A. R. M. C. (2016). Avaliação do Conhecimento de Enfermagem em Terapia Intensiva Quanto às Medidas de Precaução Padrão e Biossegurança em Hospitais Públicos na Amazônia Ocidental Brasileira [Monografia de MBA, Instituto Nacional de Ensino Superior e Pesquisa].

Gil, A. C., Bordignon, A. P. P., Castro, E. A. R., Castro, S. T., Rafael, R. M. R., \& Pereira, J. A. A. (2018). Avaliação microbiológica de superfícies em terapia intensiva: reflexões sobre as estratégias preventivas de infecções nosocomiais. Revista Enfermagem UERJ, 26, e26388. https://doi.org/10.12957/reuerj.2018.26388

Melo, M. S. (2019). Ações para a prevenção e controle da resistência bacteriana em hospitais de grande porte de Minas Gerais [Dissertação de Mestrado, Universidade Federal de Minas Gerais]. Repositório Institucional da UFMG. http://hdl.handle.net/1843/31014

Moher, D., Liberati, A., Tetzlaff, J., \& Altman, D. G., The PRISMA Group. (2009). Preferred Reporting Items for Systematic Reviews and Meta-Analyses: The PRISMA Statement. PLOS Medicine, 6(7), e1000097. https://doi.org/10.1371/journal.pmed.1000097 
Moralejo, D., El Dib, R., Prata, R. A., Barretti, P., \& Corrêa, I. (2018). Improving adherence to Standard Precautions for the control of health care-associated infections. Cochrane Database of Systematic Reviews, 2(2), CD010768. https://doi.org/10.1002/14651858.CD010768.pub2

Ndu, A. C., \& Arinze-Onyia, S. U. (2017). Standard precaution knowledge and adherence: Do Doctors differ from Medical Laboratory Scientists? Malawi medical journal: the journal of Medical Association of Malawi, 29(4), 294-300. https://doi.org/10.4314/mmj.v29i4.3

Pedrosa, K. K. A., Oliveira, I. C. M., Feijão, A. R., \& Machado, R. C. (2015). Enfermagem baseada em evidência: caracterização dos estudos no Brasil. Cogitare Enfermagem, 20(4), 733-741. https://doi.org/10.5380/ce.v20i4.40768

Piai-Morais, T. H., Orlandi, F. S., \& Figueiredo, R. M. (2015). Fatores que influenciam a adesão às precauções-padrão entre profissionais de enfermagem em hospital psiquiátrico. Revista da Escola de Enfermagem da USP, 49(3), 473-480. https://doi.org/10.1590/S0080-623420150000300016

Porto, J. S., \& Marziale, M. H. P. (2016). Motivos e consequências da baixa adesão às precauções padrão pela equipe de enfermagem. Revista Gaúcha de Enfermagem, 37(2), e57395. https://doi.org/10.1590/1983-1447.2016.02.57395

Reis, T. B., Tacla, M. T. G. M., Ferrari, R. A. P., Sant'Anna, F. L., \& Faccioli, S. C. (2015). Precaução de Contato: percepção dos acompanhantes de crianças internadas em Unidade Pediátrica. Ciência, Cuidado e Saúde, 14(3), 1315-1322. https://doi.org/10.4025/cienccuidsaude.v14i3.24171

Siegel, J. D., Rhinehart, E., Jackson, M., Chiarello, L., \& Healthcare Infection Control Practices Advisory Committee. (2019). 2007 Guideline for isolation precautions: preventing transmission of infections agents in healthcare settings. https://www.cdc.gov/infectioncontrol/pdf/guidelines/isolation-guidelinesH.pdf

Silva, B. R., Carreiro, M. A, Simões, B. F. T., \& Paula, D. G. (2018). Monitoramento da adesão à higiene das mãos em uma unidade de terapia intensiva. Revista Enfermagem UERJ, 26, e33087. https://doi.org/10.12957/reuerj.2018.33087

Whittemore, R., \& Knalf, K. (2005). The integrative review: updated methodology. Journal of Advanced Nursing, 52(5), 546-553. https://doi.org/10.1111/j.1365-2648.2005.03621.x

World Health Organization. (2009). WHO Guidelines on Hand Hygiene in Health Care: First Global Patient Safety Challenge Clean Care is Safer Care. https://www.who.int/publications/i/item/9789241597906

World Health Organization. (2016). Health care without avoidable infections: the critical role of infection prevention and control. https://apps.who.int/iris/bitstream/handle/10665/246235/WHO-HIS-SDS-2016.10-eng.pdf 